\title{
Shifts, rotations and distributional chaos
}

Dongsheng $\mathrm{Xu}^{1,2^{*}}$, Kaili Xiang ${ }^{1}$ and Shudi Liang ${ }^{2}$

"Correspondence:

xudongshengmath@126.com

'School of Economic Mathematics, Southwestern University of Finance and Economics, Chengdu, P.R. China ${ }^{2}$ School of Sciences, Southwest Petroleum University, Chengdu, P.R. China

\section{Springer}

\begin{abstract}
Let $R_{r_{0}}, R_{r_{1}}: \mathbb{S}^{1} \rightarrow \mathbb{S}^{1}$ be rotations on the unit circle $\mathbb{S}^{1}$ and define

$f: \Sigma_{2} \times \mathbb{S}^{1} \longrightarrow \Sigma_{2} \times \mathbb{S}^{1}$ as

$f(x, t)=\left(\sigma(x), R_{r_{x}}(t)\right)$,

for $x=x_{1} x_{2} \cdots \in \Sigma_{2}:=\{0,1\}^{\mathbb{N}}, t \in \mathbb{S}^{1}$, where $\sigma: \Sigma_{2} \rightarrow \Sigma_{2}$ is the shift, and $r_{0}$ and $r_{1}$ are rotational angles. It is first proved that the system $\left(\Sigma_{2} \times \mathbb{S}^{1}, f\right)$ exhibits maximal distributional chaos for any $r_{0}, r_{1} \in \mathbb{R}$ (no assumption of $r_{0}, r_{1} \in \mathbb{R} \backslash \mathbb{Q}$ ), generalizing Theorem 1 in Wu and Chen (Topol. Appl. 162:91-99, 2014). It is also obtained that $\left(\Sigma_{2} \times \mathbb{S}^{1}, f\right)$ is cofinitely sensitive and $\left(\hat{\mathscr{M}}^{1}, \hat{\mathscr{M}}^{1}\right)$-sensitive and that $\left(\Sigma_{2} \times \mathbb{S}^{1}, f\right)$ is densely chaotic if and only if $r_{1}-r_{0} \in \mathbb{R} \backslash \mathbb{Q}$.
\end{abstract}

MSC: $54 \mathrm{H} 20$

Keywords: Distributional chaos; $\mathscr{F}$-sensitivity; $\left(\mathscr{F}_{1}, \mathscr{F}_{2}\right)$-sensitivity; Dense chaos

\section{Introduction and preliminaries}

A discrete dynamical system (briefly, dynamical system) is a pair $(X, g)$, where $X$ is a compact metric space and $g: X \longrightarrow X$ is a continuous map. Let $\mathbb{N}=\{1,2,3, \ldots\}$ and $\mathbb{Z}^{+}=\{0,1,2, \ldots\}$. Sharkovsky's amazing discovery [10], as well as Li and Yorke's famous work which introduced the concept of "chaos" known as Li-Yorke chaos today in a mathematically rigorous way [4], have activated sustained interest and provoked the recent rapid advancement of the frontier research on discrete chaos theory. In their study, Li and Yorke suggested considering "divergent pairs" $(x, y)$, which are proximal but not asymptotic and called $L i$-Yorke pairs, i.e.,

$$
\liminf _{n \rightarrow \infty} d\left(g^{n}(x), g^{n}(y)\right)=0 \quad \text { and } \quad \limsup _{n \rightarrow \infty} d\left(g^{n}(x), g^{n}(y)\right)>0
$$

A pair $(x, y)$ is called a Li-Yorke pair of modulus $\delta$ if

$$
\liminf _{n \rightarrow \infty} d\left(g^{n}(x), g^{n}(y)\right)=0 \text { and } \quad \limsup _{n \rightarrow \infty} d\left(g^{n}(x), g^{n}(y)\right)>\delta
$$

Clearly, $(x, y)$ is a Li-Yorke pair if and only if it is a Li-Yorke pair of modulus $\delta$ for some $\delta>0$. The set of Li-Yorke pairs of modulus $\delta$ is denoted by $\mathrm{LY}(g, \delta)$ and the set of $\mathrm{Li}-$ Yorke pairs by $\mathrm{LY}(\mathrm{g})$. According to the idea of Li and Yorke [4], a subset $D$ of $X$ is a $\delta$ scrambled set of $g$, if $D \times D \backslash \Delta \subset \mathrm{LY}(g, \delta)$, where $\Delta=\{(x, x) \in X \times X: x \in X\}$. In particular,

(c) The Author(s) 2019. This article is distributed under the terms of the Creative Commons Attribution 4.0 International License (http://creativecommons.org/licenses/by/4.0/), which permits unrestricted use, distribution, and reproduction in any medium, provided you give appropriate credit to the original author(s) and the source, provide a link to the Creative Commons license, and indicate if changes were made. 
if $D \times D \backslash \Delta \subset \operatorname{LY}(g)$, then $D$ is a scrambled set of $g$. If a scrambled set $D$ of $g$ is also uncountable, it is called a Li-Yorke scrambled set for $g$, and $g$ is said to be chaotic in the sense of Li-Yorke, or Li-Yorke chaotic.

In 1985, Piórek [8] introduced the concept of generic chaos. Inspired by this, Snoha [11, 12] defined generic $\delta$-chaos, dense chaos, and dense $\delta$-chaos in 1990. The notion of $\mathrm{Li}-$ Yorke sensitivity was firstly introduced by Akin and Kolyada [1] in 2003. More recent results on chaos can be found in $[2,3,7,17,18,20-31]$.

Definition $1([1,8,11,12])$ A dynamical system $(X, g)$ is

(1) sensitive if there exists $\varepsilon>0$ such that for any $x \in X$ and any $\delta>0$, there exist $y \in B(x, \delta):=\{z \in X: d(z, x)<\delta\}$ and $n \in \mathbb{N}$, such that $d\left(g^{n}(x), g^{n}(y)\right) \geq \varepsilon ;$

(2) Li-Yorke sensitive if there exists $\varepsilon>0$ such that for any $x \in X$ and any $\delta>0$, there exists $y \in B(x, \delta)$ such that $(x, y) \in \operatorname{LY}(g, \varepsilon)$;

(3) densely chaotic if $\mathrm{LY}(g)$ is dense in $X \times X$;

(4) densely $\delta$-chaotic for some $\delta>0$ if $\operatorname{LY}(g, \delta)$ is dense in $X \times X$;

(5) generically chaotic if $\operatorname{LY}(g)$ is residual in $X \times X$;

(6) generically $\delta$-chaotic for some $\delta>0$ if $\operatorname{LY}(g, \delta)$ is residual in $X \times X$.

Distributional chaos. The notion of distributional chaos was first introduced in [9], where it was called "strong chaos", which is characterized by a distributional function of distances between trajectories of two points. It is described as follows.

Let $(X, g)$ be a dynamical system. For any pair $(x, y) \in X \times X$, define the lower and upper distributional functions as

$$
F_{x, y}(t, g)=\liminf _{n \rightarrow \infty} \frac{1}{n}\left|\left\{i: d\left(g^{i}(x), g^{i}(y)\right)<t, 0 \leq i<n\right\}\right|
$$

and

$$
F_{x, y}^{*}(t, g)=\limsup _{n \rightarrow \infty} \frac{1}{n}\left|\left\{i: d\left(g^{i}(x), g^{i}(y)\right)<t, 0 \leq i<n\right\}\right|,
$$

respectively, where $|A|$ denotes the cardinality of set $A$. Both functions $F_{x, y}$ and $F_{x, y}^{*}$ are non-decreasing and $F_{x, y} \leq F_{x, y}^{*}$.

According to Schweizer and Smítal [9], a dynamical system $(X, g)$ is distributionally $\varepsilon$ chaotic for some $\varepsilon>0$ if there exists an uncountable subset $\mathcal{S} \subset X$ such that for any pair of distinct points $x, y \in \mathcal{S}$, one has that $F_{x, y}^{*}(t, g)=1$ for all $t>0$ and $F_{x, y}(\varepsilon, g)=0$. The set $\mathcal{S}$ is called a distributionally $\varepsilon$-scrambled set and the pair $(x, y)$ a distributionally $\varepsilon$-chaotic pair. If $(X, g)$ is distributionally $\varepsilon$-chaotic for any $0<\varepsilon<\operatorname{diam}(X)$, then $(X, g)$ is said to exhibit maximal distributional chaos.

Let $\mathcal{P}$ be the collection of all subsets of $\mathbb{Z}^{+}$. A collection $\mathscr{F} \subset \mathcal{P}$ is called a Furstenberg family if it is hereditary upwards, i.e., $F_{1} \subset F_{2}$ and $F_{1} \in \mathscr{F}$ imply $F_{2} \in \mathscr{F}$. A family $\mathscr{F}$ is proper if it is a proper subset of $\mathcal{P}$, i.e., neither empty nor the whole $\mathcal{P}$. It is easy to see that $\mathscr{F}$ is proper if and only if $\mathbb{Z}^{+} \in \mathscr{F}$ and $\emptyset \notin \mathscr{F}$. Let $\mathscr{F}$ inf be the collection of all infinite subsets of $\mathbb{Z}^{+}$and $\mathscr{F}_{c f}$ the family of cofinite subset, i.e., the collection of subsets of $\mathbb{Z}^{+}$with finite complements.

For $A \subset \mathbb{Z}^{+}$, define

$$
\bar{d}(A)=\limsup _{n \rightarrow \infty} \frac{1}{n}|A \cap[0, n-1]| \quad \text { and } \quad \underline{d}(A)=\liminf _{n \rightarrow \infty} \frac{1}{n}|A \cap[0, n-1]| .
$$


Then, $\bar{d}(A)$ and $\underline{d}(A)$ are the upper density and the lower density of $A$, respectively. Fix any $\alpha \in[0,1]$ and denote by $\hat{\mathscr{M}}_{\alpha}\left(\right.$ resp. $\left.\hat{\mathscr{M}}^{\alpha}\right)$ the family consisting of sets $A \subset \mathbb{Z}^{+}$with $\underline{d}(A) \geq \alpha$ (resp. $\bar{d}(A) \geq \alpha$ ).

Using Furstenberg family, Wang et al. $[6,13,14]$ introduced the notions of $\mathscr{F}$-sensitivity, $\left(\mathscr{F}_{1}, \mathscr{F}_{2}\right)$-sensitivity, and $\left(\mathscr{F}_{1}, \mathscr{F}_{2}\right)$-chaos for generalizing sensitivity, Li-Yorke sensitivity, and Li-Yorke chaos, respectively.

Let $\mathscr{F}$ be a Furstenberg family. According to Moothathu [6], a dynamical system $(X, g)$ is

(1) $\mathscr{F}$-sensitive if there exists $\varepsilon>0$ such that for any nonempty open subset $U$ of $X$, $\left\{n \in \mathbb{Z}^{+}: \operatorname{diam}\left(g^{n}(U)\right) \geq \delta\right\} \in \mathscr{F}$.

(2) cofinitely sensitive if there exists $\varepsilon>0$ such that for any nonempty open subset $U$ of $X,\left\{n \in \mathbb{Z}^{+}: \operatorname{diam}\left(g^{n}(U)\right) \geq \delta\right\} \in \mathscr{F}_{c f}$.

Definition $2([13,14])$ Let $\mathscr{F}_{1}, \mathscr{F}_{2}$ be Furstenberg families. A dynamical system $(X, g)$ is

(1) $\left(\mathscr{F}_{1}, \mathscr{F}_{2}\right)$-sensitive if there exists some $\varepsilon>0$ such that for any $x \in X$ and any $\delta>0$, there exists $y \in B(x, \delta)$ such that $\left\{n \in \mathbb{Z}^{+}: d\left(g^{n}(x), g^{n}(y)\right)<t\right\} \in \mathscr{F}_{1}$ for any $t>0$ and $\left\{n \in \mathbb{Z}^{+}: d\left(g^{n}(x), g^{n}(y)\right)>\varepsilon\right\} \in \mathscr{F}_{2}$.

(2) $\left(\mathscr{F}_{1}, \mathscr{F}_{2}\right)$-chaotic if there exists an uncountable subset $D \subset X$ such that for any $(x, y) \in D \times D \backslash \Delta$, there exists $\delta>0$ such that $\left\{n \in \mathbb{Z}^{+}: d\left(g^{n}(x), g^{n}(y)\right)<t\right\} \in \mathscr{F}_{1}$ for any $t>0$ and $\left\{n \in \mathbb{Z}^{+}: d\left(g^{n}(x), g^{n}(y)\right)>\delta\right\} \in \mathscr{F}_{2}$.

From Definition 2, it can be verified that a dynamical system is Li-Yorke chaotic (resp., distributionally chaotic, Li-Yorke sensitive) if and only if it is $\left(\mathscr{F}_{\text {inf }}, \mathscr{F}_{\text {inf }}\right)$-chaotic (resp., $\left(\hat{\mathscr{M}}^{1}, \hat{\mathscr{M}}^{1}\right)$-chaotic, $\left(\mathscr{F}_{\text {inf }}, \mathscr{F}_{\text {inf }}\right)$-sensitive $)$.

Shift and rotation. Let $\Sigma=\{0,1\}$ and consider a product space $\Sigma_{2}=\Sigma^{\mathbb{N}}$ with the product topology which is compact and metrizable. Let $\Sigma_{2}$ endow with the following metric:

$$
d_{1}(x, y)= \begin{cases}0, & x=y, \\ \frac{1}{\min \left\{m \geq 1: x_{m} \neq y_{m}\right\}}, & x \neq y,\end{cases}
$$

for any $x=x_{1} x_{2} \cdots, y=y_{1} y_{2} \cdots \in \Sigma_{2}$.

Define the shift $\sigma: \Sigma_{2} \longrightarrow \Sigma_{2}$ by $\sigma(x)=x_{2} x_{3} \cdots$ for any $x=x_{1} x_{2} \cdots \in \Sigma_{2}$. Clearly, $\sigma$ is continuous. If $X$ is a closed and invariant subset of $\Sigma_{2}$, then $\left(X,\left.\sigma\right|_{X}\right)$ is called a shift space or subshift.

Any element $A$ of the set $\Sigma^{n}$ is called an $n$-word over $\Sigma$ and the length of $A$ is $n$, denoted by $|A|$. A word over $\Sigma$ is an element of the set $\bigcup_{n \in \mathbb{N}} \Sigma^{n}$. Let $A=a_{1} \cdots a_{n} \in \Sigma^{n}$ and $B=$ $b_{1} \cdots b_{m} \in \Sigma^{m}$. Denote $A B=a_{1} \cdots a_{n} b_{1} \cdots b_{m}$ and $\bar{A}=\overline{a_{1}} \cdots \overline{a_{n}}$, where

$$
\overline{a_{i}}= \begin{cases}0, & a_{i}=1, \\ 1, & a_{i}=0 .\end{cases}
$$

Clearly, $A B \in \Sigma^{n+m}$ and $\bar{A} \in \Sigma^{n}$. For any $a \in \Sigma$, denote $a^{n}$ as an $n$-length concatenation of $a$ (for example, $\left.0^{3}=000\right)$, and $a^{\infty}=a a \cdots$ as an infinite concatenation. If $x=x_{1} x_{2} \cdots \in \Sigma_{2}$ and $i \leq j \in \mathbb{N}$, then let $x_{[i, j]}=x_{i} x_{i+1} \cdots x_{j}$ and $x_{(i, j]}=x_{[i+1, j]}$. For any $B=b_{1} \cdots b_{n} \in \bigcup_{n \in \mathbb{N}} \Sigma^{n}$, the set $[B]=\left\{x_{1} x_{2} \cdots \in \Sigma_{2}: x_{i}=b_{i}, 1 \leq i \leq n\right\}$ is called the cylinder generated by $B$. For any $n \in \mathbb{N}$, let $\mathcal{B}_{n}=\left\{\left[b_{1} \cdots b_{n}\right]: b_{i} \in \Sigma, 1 \leq i \leq n\right\}$. 
The function $\wp: \bigcup_{n \in \mathbb{N}} \Sigma^{n} \longrightarrow \mathbb{Z}^{+}$is defined by

$$
\wp(A)=|A|-\sum_{j=1}^{|A|} a_{j}=\left|\left\{j: 1 \leq j \leq|A|, a_{j}=0\right\}\right|,
$$

for any $A=a_{1} \cdots a_{|A|} \in \bigcup_{n \in \mathbb{N}} \Sigma^{n}$.

Consider the unit circle $\mathbb{S}^{1}$ defined by

$$
\mathbb{S}^{1}=\left\{(x, y): x^{2}+y^{2}=1\right\} \subset \mathbb{R}^{2}
$$

Identifying $\mathbb{R}^{2}$ with the complex plane $\mathbb{C}$, one can write

$$
\mathbb{S}^{1}=\left\{e^{2 \pi i \theta}: 0 \leq \theta<1\right\} \subset \mathbb{C} .
$$

Consider a rotation $R_{r}$ of angle $2 \pi r$ on the circle, given by

$$
R_{r}\left(e^{2 \pi i \theta}\right)=e^{2 \pi i(\theta+r)}
$$

There is a natural distance $d_{2}\left(z_{1}, z_{2}\right)$ between points $z_{1}$ and $z_{2}$ on $\mathbb{S}^{1}$, given by the arc length distance. It is normalized by dividing with $2 \pi$.

This paper considers the following dynamical system.

Let $R_{r_{0}}, R_{r_{1}}: \mathbb{S}^{1} \longrightarrow \mathbb{S}^{1}$ be rotations and define $f: \Sigma_{2} \times \mathbb{S}^{1} \longrightarrow \Sigma_{2} \times \mathbb{S}^{1}$ by

$$
f(x, t)=\left(\sigma(x), R_{r_{x_{1}}}(t)\right),
$$

for any $x=x_{1} x_{2} \cdots \in \Sigma_{2}, t \in \mathbb{S}^{1}$ (so, $r_{x_{1}}=r_{0}$ or $r_{1}$ ). Note that the $n$th iteration of $f$ at the point $(x, t) \in \Sigma_{2} \times \mathbb{S}^{1}$ is given by

$$
f^{n}(x, t)=\left(\sigma^{n}(x), R_{r_{x_{n}}} \circ \cdots \circ R_{r_{x_{2}}} \circ R_{r_{x_{1}}}(t)\right) .
$$

Let $d$ be the product metric on the product space $\Sigma_{2} \times \mathbb{S}^{1}$, i.e.,

$$
d\left(\left(x, t_{1}\right),\left(y, t_{2}\right)\right)=\max \left\{d_{1}(x, y), d_{2}\left(t_{1}, t_{2}\right)\right\},
$$

for any $\left(x, t_{1}\right),\left(y, t_{2}\right) \in \Sigma_{2} \times \mathbb{S}^{1}$.

Wang et al. [15] proved that a dynamical system having a regular shift-invariant set is distributionally chaotic and posed the following question:

Question 3 ([15]) Is the system $\left(\Sigma_{2} \times \mathbb{S}^{1}, f\right)$ distributionally chaotic when $r_{0}, r_{1} \in \mathbb{R} \backslash \mathbb{Q}$ with $r_{0} \neq \pm r_{1}$ ?

Recently, Wu and Chen [19] gave a positive answer to this question and proved that $\left(\Sigma_{2} \times \mathbb{S}^{1}, f\right)$ is Li-Yorke sensitive. By further discussing $\left(\Sigma_{2} \times \mathbb{S}^{1}, f\right)$, we in this paper investigate the chaos of $\left(\Sigma_{2} \times \mathbb{S}^{1}, f\right)$ for any $r_{0}, r_{1} \in \mathbb{R}$ (not assuming $r_{0}, r_{1} \in \mathbb{R} \backslash \mathbb{Q}$ ). More precisely, we first prove that $\left(\Sigma_{2} \times \mathbb{S}^{1}, f\right)$ is distributionally chaotic for any $r_{0}, r_{1} \in \mathbb{R}$. Meanwhile, we obtain that (1) it is cofinitely sensitive and $\left(\hat{\mathscr{M}}^{1}, \hat{\mathscr{M}}^{1}\right)$-sensitive, and (2) it is densely chaotic if and only if $r_{1}-r_{0} \in \mathbb{R} \backslash \mathbb{Q}$. 


\section{Distributional chaos for $\left(\Sigma_{2} \times \mathbb{S}^{1}, f\right)$}

The following lemma will be the key to prove the distributional chaoticity for $\left(\Sigma_{2} \times \mathbb{S}^{1}, f\right)$.

Lemma $4([5,16])$ There exists an uncountable subset $E$ of $\Sigma_{2}$ such that for any distinct points $x=x_{1} x_{2} \cdots, y=y_{1} y_{2} \cdots$ in $E$, they satisfy $x_{n}=y_{n}$ for infinitely many $n$ and $x_{m} \neq y_{m}$ for infinitely many $m$.

Theorem 5 There exists an uncountable subset $\mathcal{T} \subset \Sigma_{2} \times \mathbb{S}^{1}$ which is a distributionally $\beta$-scrambled set of for any $0<\beta \leq \operatorname{diam}\left(\Sigma_{2} \times \mathbb{S}^{1}\right)=1$, i.e., $\left(\Sigma_{2} \times \mathbb{S}^{1}, f\right)$ exhibits maximal distributional chaos.

Proof Let $\mathscr{L}_{1}=L_{1}=2$ and $L_{k+1}=2^{\mathscr{L}_{k}}+2 \mathscr{L}_{k}, \mathscr{L}_{k+1}=\mathscr{L}_{k}+L_{k+1}$ for any $k \in \mathbb{N}$. From Lemma 4, it follows that there exists an uncountable subset $E \subset \Sigma_{2}$ such that for any two distinct points $x=x_{1} x_{2} \cdots, y=y_{1} y_{2} \cdots \in E, x_{n}=y_{n}$ holds for infinitely many $n$ and $x_{m} \neq y_{m}$ holds for infinitely many $m$. For any $x=x_{1} x_{2} \cdots \in E$, take $\tilde{x}=\widetilde{x}_{1} \tilde{x}_{2} \tilde{x}_{3} \cdots$ as

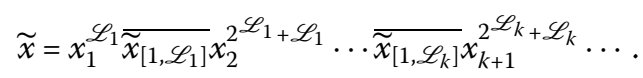

Set $\mathcal{T}=\{\tilde{x}: x \in E\} \times\{1\}$. Clearly, $\mathcal{T}$ is uncountable.

Now, we claim that $\mathcal{T}$ is a distributionally $\beta$-scrambled set of $f$ for any $0<\beta \leq 1$.

Fixing any pair of distinct points $(x, 1),(y, 1) \in \mathcal{T}$, it follows from the construction of of $\mathcal{T}$ that there exist two different points $u=u_{1} u_{2} \cdots, v=v_{1} v_{2} \cdots \in E$ and two increasing sequences $\left\{p_{k}\right\}_{k=1}^{\infty},\left\{q_{k}\right\}_{k=1}^{\infty}$ of $\mathbb{N}$ such that for any $k \in \mathbb{N}$,

(a) $\tilde{u}=x, \widetilde{v}=y$,

(b) $u_{p_{k}}=v_{p_{k}}, u_{q_{k}}=\overline{v_{q_{k}}}$.

(1) Note that $\wp\left(\widetilde{u}_{\left[1,2 \mathscr{L}_{p_{k}}\right]}\right)=\wp\left(\widetilde{v}_{\left[1,2 \mathscr{L}_{p_{k}}\right]}\right)$ for any $k \in \mathbb{N}$. This implies that

$$
R_{r_{x_{2} \mathscr{L}_{p_{k}}}} \circ \cdots \circ R_{r_{x_{2}}} \circ R_{r_{x_{1}}}=R_{r_{y_{2} \mathscr{P}_{p_{k}}}} \circ \cdots \circ R_{r_{y_{2}}} \circ R_{r_{y_{1}}} .
$$

For any $2 \mathscr{L}_{p_{k}} \leq j \leq \mathscr{L}_{p_{k}+1}-\mathscr{L}_{p_{k}}$, the first $\mathscr{L}_{p_{k}}$ respective symbols of $\sigma^{j}(x)$ and $\sigma^{j}(y)$ coincide, implying that

$$
d_{1}\left(\sigma^{j}(x), \sigma^{j}(y)\right) \leq \frac{1}{\mathscr{L}_{p_{k}}} \rightarrow 0 \quad(k \rightarrow \infty)
$$

Meanwhile, applying (2.1) yields that for any $2 \mathscr{L}_{p_{k}} \leq j \leq \mathscr{L}_{p_{k}+1}-\mathscr{L}_{p_{k}}$,

$$
R_{r_{x_{j}}} \circ \cdots \circ R_{r_{x_{2}}} \circ R_{r_{x_{1}}}(1)=R_{r_{y_{j}}} \circ \cdots \circ R_{r_{y_{2}}} \circ R_{r_{y_{1}}}(1) .
$$

Then, for any $t>0$, there exists some $K \in \mathbb{N}$ such that for any $k \geq K$ and any $2 \mathscr{L}_{p_{k}} \leq j \leq$ $\mathscr{L}_{p_{k}+1}-\mathscr{L}_{p_{k}}$,

$$
d\left(f^{j}(x, 1), f^{j}(y, 1)\right)<t .
$$

Consequently,

$$
F_{(x, 1),(y, 1)}^{*}(t, f)
$$




$$
\begin{aligned}
& =\limsup _{n \rightarrow \infty} \frac{1}{n}\left|\left\{k: d\left(f^{k}(x, 1), f^{k}(y, 1)\right)<t, 0 \leq k<n\right\}\right| \\
& \geq \limsup _{k \rightarrow \infty} \frac{1}{\mathscr{L}_{p_{k}+1}-\mathscr{L}_{p_{k}}}\left|\left\{n: d\left(f^{j}(x, 1), f^{j}(y, 1)\right)<t, 0 \leq j<\mathscr{L}_{p_{k}+1}-\mathscr{L}_{p_{k}}\right\}\right| \\
& \geq \limsup _{k \rightarrow \infty} \frac{\mathscr{L}_{p_{k}+1}-3 \mathscr{L}_{p_{k}}}{\mathscr{L}_{p_{k}+1}-\mathscr{L}_{p_{k}}} \\
& =\limsup _{k \rightarrow \infty} \frac{2^{\mathscr{L}_{p_{k}}}}{2^{\mathscr{L}_{p_{k}}+2 \mathscr{L}_{p_{k}}}} \\
& =1 .
\end{aligned}
$$

(2) It is easy to see that for any $2 \mathscr{L}_{q_{k}} \leq j \leq \mathscr{L}_{q_{k+1}}-1$, the first respective symbols of $\sigma^{j}(x)$ and $\sigma^{j}(y)$ are distinct, implying that $d_{1}\left(\sigma^{j}(x), \sigma^{j}(y)\right)=1$. Then, for any $2 \mathscr{L}_{q_{k}} \leq j \leq \mathscr{L}_{q_{k}+1}-1$ and any $0<\beta \leq 1$,

$$
d\left(f^{j}(x, 1), f^{j}(y, 1)\right) \geq \beta
$$

Therefore,

$$
\begin{aligned}
& F_{(x, 1),(y, 1)}(\beta, f) \\
& \quad=\liminf _{n \rightarrow \infty} \frac{1}{n}\left|\left\{k: d\left(f^{k}(x, 1), f^{k}(y, 1)\right)<\beta, 0 \leq k<n\right\}\right| \\
& \quad \leq \liminf _{k \rightarrow \infty} \frac{1}{\mathscr{L}_{q_{k}+1}-1}\left|\left\{j: d\left(f^{k j}(x, 1), f^{j}(y, 1)\right)<\beta, 0 \leq j \leq \mathscr{L}_{q_{k}+1}-1\right\}\right| \\
& \quad \leq \liminf _{k \rightarrow \infty} \frac{2 \mathscr{L}_{q_{k}}}{\mathscr{L}_{q_{k}+1}-1} \\
& \quad=\liminf _{k \rightarrow \infty} \frac{2 \mathscr{L}_{q_{k}}}{3 \mathscr{L}_{q_{k}}+2^{\mathscr{L}_{q_{k}}}} \\
& \quad=0 .
\end{aligned}
$$

Hence, $\mathcal{T}$ is a distributionally $\beta$-scrambled set for any $0<\beta \leq 1$, i.e., $\left(\Sigma_{2} \times \mathbb{S}^{1}, f\right)$ exhibits maximal distributional chaos.

\section{Other chaos for $\left(\Sigma_{2} \times \mathbb{S}^{1}, f\right)$}

This section shall show that $\left(\Sigma_{2} \times \mathbb{S}^{1}, f\right)$ is cofinitely sensitive and $\left(\hat{\mathscr{M}}^{1}, \hat{\mathscr{M}}^{1}\right)$-sensitive and obtain a sufficient and necessary condition for a dense chaos.

Theorem 6 The system $\left(\Sigma_{2} \times \mathbb{S}^{1}, f\right)$ is cofinitely sensitive.

Proof Given any point $(x, t) \in \Sigma_{2} \times \mathbb{S}^{1}$ and any $\delta>0$, take $K=\left[\frac{1}{\delta}\right]+2$ and assume that $x=x_{1} x_{2} x_{3} \cdots$. Choose $y=y_{1} y_{2} y_{3} \cdots \in \Sigma_{2}$ by the following formula:

$$
y_{n}= \begin{cases}x_{n}, & 1 \leq n \leq K, \\ \overline{x_{n}}, & n>K .\end{cases}
$$


Clearly, $d((x, t),(y, t))<\delta$. For any $n>K$, it can be verified that

$$
d\left(f^{n}(x, t), f^{n}(y, t)\right) \geq d_{1}\left(\sigma^{n}(x), \sigma^{n}(y)\right)=1
$$

implying that $\left(\Sigma_{2} \times \mathbb{S}^{1}, f\right)$ is cofinitely sensitive, due to the arbitrariness of $\delta$ and $(x, t)$

Theorem 7 The system $\left(\Sigma_{2} \times \mathbb{S}^{1}, f\right)$ is $\left(\hat{\mathscr{M}}^{1}, \hat{\mathscr{M}}^{1}\right)$-sensitive. In particular, it is Li-Yorke sensitive.

Proof Given any $(x, t) \in \Sigma_{2} \times \mathbb{S}^{1}$ and any $\delta>0$, choose $L_{1}=\mathscr{L}_{1}=\left[\frac{1}{\delta}\right]+2$ and $L_{k+1}=2^{\mathscr{L}_{k}}+$ $2 \mathscr{L}_{k}, \mathscr{L}_{k+1}=\mathscr{L}_{k}+L_{k+1}$ for any $k \in \mathbb{N}$. Take $y=y_{1} y_{2} y_{3} \cdots \in \Sigma_{2}$ as the following inductive method:

(1) $y_{\left[1, \mathscr{L}_{2}\right]}=x_{\left[1, \mathscr{L}_{1}\right]} \overline{\bar{x}_{\left[1, \mathscr{L}_{1}\right]}} x_{\left[2 \mathscr{L}_{1}+1, \mathscr{L}_{2}\right]}$

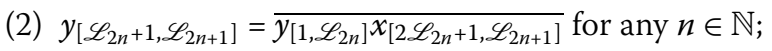

(3) $y_{\left[\mathscr{L}_{2 n+1}+1, \mathscr{L}_{2 n+2}\right]}=\bar{y}_{\left[1, \mathscr{L}_{2 n+1}\right]} x_{\left[2 \mathscr{L}_{2 n+1}+1, \mathscr{L}_{2 n+2}\right]}$ for any $n \in \mathbb{N}$.

Clearly, $d((x, t),(y, t))=d_{1}(x, y)=\frac{1}{\mathscr{L}_{1}+1}<\delta$. Similarly as in the proof of Theorem 5 , it can be verified that

(1) for any $\varepsilon>0,\left\{n \in \mathbb{Z}^{+}: d\left(f^{n}(x, t), f^{n}(y, t)\right)<t\right\} \in \hat{\mathscr{M}}^{1}$;

(2) for any $0<\beta \leq 1,\left\{n \in \mathbb{Z}^{+}: d\left(f^{n}(x, t), f^{n}(y, t)\right) \geq \beta\right\} \in \hat{\mathscr{M}}^{1}$, implying that $\left(\Sigma_{2} \times \mathbb{S}^{1}, f\right)$ is $\left(\hat{\mathscr{M}}^{1}, \hat{\mathscr{M}}^{1}\right)$-sensitive, due to the arbitrariness of $\delta$ and $(x, t)$.

Theorem 8 The system $\left(\Sigma_{2} \times \mathbb{S}^{1}, f\right)$ is densely chaotic if and only if $r_{1}-r_{0} \in \mathbb{R} \backslash \mathbb{Q}$.

Proof $(\Longrightarrow)$ Suppose on the contrary that $r_{1}-r_{0} \in \mathbb{Q}$. Then there exist $t_{0} \in \mathbb{S}^{1}$ and $\eta>0$ such that

$$
\xi:=\inf \left\{d_{2}\left(z_{1}, z_{2}\right): z_{1} \in B\left(t_{0}, \eta\right), z_{2} \in \bigcup_{n \in \mathbb{Z}} R_{r_{1}-r_{0}}^{n}(B(1, \eta))\right\}>0
$$

Fix any $x \in \Sigma_{2}$ and set $U=B(x, \eta) \times B(1, \eta)$ and $V=B(x, \eta) \times B\left(t_{0}, \eta\right)$. Clearly, both $U$ and $V$ are nonempty open subsets of $\Sigma_{2} \times \mathbb{S}^{1}$.

Now, we claim that $(U \times V) \cap \operatorname{LY}(f)=\emptyset$.

In fact, for any $\left(\left(y, t_{1}\right),\left(z, t_{2}\right)\right) \in U \times V$ and any $n \in \mathbb{Z}^{+}$, it can be verified that for any $n \in \mathbb{Z}^{+}$,

$$
\begin{aligned}
R_{r_{y_{n}}} & \circ \cdots \circ R_{r_{y_{2}}} \circ R_{r_{y_{1}}}\left(t_{1}\right) \\
\quad= & e^{2 \pi i\left(\sum_{k=1}^{n} r_{y_{k}}\right)} t_{1}=e^{2 \pi i\left[r_{0} \wp\left(y_{[1, n]}\right)+r_{1}\left(n-\wp\left(y_{[1, n]}\right)\right)\right]} t_{1} \\
= & e^{2 \pi i\left[r_{0} \wp\left(z_{[1, n]}\right)+r_{1}\left(n-\wp\left(z_{[1, n]}\right)\right)\right]} \cdot e^{2 \pi i\left[\left(r_{1}-r_{0}\right)\left(\wp\left(z_{[1, n]}\right)-\wp\left(y_{[1, n]}\right)\right)\right]} t_{1}
\end{aligned}
$$

and

$$
R_{r_{z_{n}}} \circ \cdots \circ R_{r_{z_{2}}} \circ R_{r_{z_{1}}}\left(t_{2}\right)=e^{2 \pi i\left(\sum_{k=1}^{n} r_{z_{k}}\right)} t_{2}=e^{2 \pi i\left[r_{0} \wp\left(z_{[1, n]}\right)+r_{1}\left(n-\wp\left(z_{[1, n]}\right)\right)\right]} t_{2},
$$

implying that

$$
d_{2}\left(R_{r_{y_{n}}} \circ \cdots \circ R_{r_{y_{2}}} \circ R_{r_{y_{1}}}\left(t_{1}\right), R_{r_{z_{n}}} \circ \cdots \circ R_{r_{2}} \circ R_{r_{z_{1}}}\left(t_{2}\right)\right)
$$




$$
\begin{aligned}
& =d_{2}\left(e^{2 \pi i\left[\left(r_{1}-r_{0}\right)\left(\wp\left(z_{[1, n]}\right)-\wp\left(y_{[1, n]}\right)\right)\right]} t_{1}, t_{2}\right) \\
& =d_{2}\left(R_{r_{1}-r_{0}}^{\wp\left(z_{[1, n]}\right)-\wp\left(y_{[1, n]}\right)}\left(t_{1}\right), t_{2}\right) \geq \xi .
\end{aligned}
$$

Therefore,

$$
\begin{aligned}
& \liminf _{n \rightarrow \infty} d\left(f^{n}\left(y, t_{1}\right), f^{n}\left(z, t_{2}\right)\right) \\
& \quad \geq \liminf _{k \rightarrow \infty} d_{2}\left(R_{r_{y_{n}}} \circ \cdots \circ R_{r_{y_{2}}} \circ R_{r_{y_{1}}}\left(t_{1}\right), R_{r_{z_{n}}} \circ \cdots \circ R_{r_{z_{2}}} \circ R_{r_{z_{1}}}\left(t_{2}\right)\right) \geq \xi>0 .
\end{aligned}
$$

This means that $(U \times V) \cap \operatorname{LY}(f)=\emptyset$. Hence, $f$ is not densely chaotic, which is a contradiction.

$(\Longleftarrow)$ For any nonempty open subsets $U, V$ of $\Sigma_{2} \times \mathbb{S}^{1}$, choose $\left(u, t_{1}\right) \in U,\left(v, t_{2}\right) \in V$ and $K \in \mathbb{N}$ such that $B\left(\left(u, t_{1}\right), \frac{1}{K}\right) \subset U$ and $B\left(\left(v, t_{2}\right), \frac{1}{K}\right) \subset V$. From $r_{1}-r_{0} \in \mathbb{R} \backslash \mathbb{Q}$, it follows that there exists an increasing sequence $\left\{p_{n}\right\}_{n=1}^{\infty} \subset \mathbb{N}$ such that $d_{2}\left(R_{r_{1}-r_{0}}^{p_{n}}\left(t_{1}\right), t_{2}\right)<\frac{1}{n}$ for any $n \in$ $\mathbb{N}$. Let $L_{1}=\mathscr{L}_{1}=K$ and $L_{n+1}=2 \mathscr{L}_{n}+p_{n}, \mathscr{L}_{n+1}=\mathscr{L}_{n}+L_{n}$ for any $n \in \mathbb{N}$. Take $\hat{u}=\hat{u}_{1} \hat{u}_{2} \hat{u}_{3} \ldots$ and $\hat{v}=\hat{v}_{1} \hat{v}_{2} \hat{v}_{3} \cdots \in \Sigma_{2}$ as

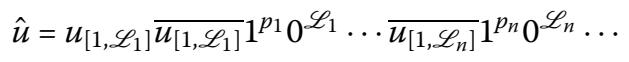

and

$$
\hat{v}=v_{\left[1, \mathscr{L}_{1}\right]} \overline{\nu_{\left[1, \mathscr{L}_{1}\right]}} 0^{p_{1}+\mathscr{L}_{1}} \ldots \overline{v_{\left[1, \mathscr{L}_{n}\right]}} 0^{p_{n}+\mathscr{L}_{n}} \ldots
$$

respectively. Clearly, $\left(\hat{u}, t_{1}\right) \in U$ and $\left(\hat{v}, t_{2}\right) \in V$.

(1) Noting that $\hat{u}_{2 \mathscr{L}_{n}+1}=1$ and $\hat{v}_{2 \mathscr{L}_{n}+1}=0$, it follows that

$$
d\left(f^{2 \mathscr{L}_{n}}\left(\hat{u}, t_{1}\right), f^{2 \mathscr{L}_{n}}\left(\hat{v}, t_{2}\right)\right) \geq d_{1}\left(\sigma^{2 \mathscr{L}_{n}}(\hat{u}), \sigma^{2 \mathscr{L}_{n}}(\hat{v})\right)=1
$$

implying that

$$
\limsup _{n \rightarrow \infty} d\left(f^{n}\left(\hat{u}, t_{1}\right), f^{n}\left(\hat{v}, t_{2}\right)\right) \geq \limsup _{n \rightarrow \infty} d\left(f^{2 \mathscr{L}_{n}}\left(\hat{u}, t_{1}\right), f^{2 \mathscr{L}_{n}}\left(\hat{v}, t_{2}\right)\right) \geq 1
$$

(2) From the fact that the first $\mathscr{L}_{n}$ respective symbols of $\sigma^{2 \mathscr{L}_{n}+p_{n}}(\hat{u})$ and $\sigma^{2 \mathscr{L}_{n}+p_{n}}(\hat{v})$ coincide, it follows that

$$
d_{1}\left(\sigma^{2 \mathscr{L}_{n}+p_{n}}(\hat{u}), \sigma^{2 \mathscr{L}_{n}+p_{n}}(\hat{v})\right) \leq \frac{1}{\mathscr{L}_{n}}<\frac{1}{n}
$$

Meanwhile, it can verified that

$$
d_{2}\left(R_{\hat{u}_{2} \mathscr{L}_{n}+p_{n}} \circ \cdots \circ R_{\hat{u}_{2}} \circ R_{\hat{u}_{1}}\left(t_{1}\right), R_{\hat{v}_{2} \mathscr{L}_{n}+p_{n}} \circ \cdots \circ R_{\hat{v}_{2}} \circ R_{\hat{v}_{1}}\left(t_{2}\right)\right)=d_{2}\left(R_{r_{1}-r_{0}}^{p_{n}}\left(t_{1}\right), t_{2}\right)<\frac{1}{n} .
$$

This, together with (3.1), implies that

$$
d\left(f^{2 \mathscr{L}_{n}+p_{n}}\left(\hat{u}, t_{1}\right), f^{2 \mathscr{L}_{n}+p_{n}}\left(\hat{v}, t_{2}\right)\right)<\frac{1}{n}
$$


Then,

$$
\liminf _{n \rightarrow \infty} d\left(f^{n}\left(\hat{u}, t_{1}\right), f^{n}\left(\hat{v}, t_{2}\right)\right) \leq \liminf _{n \rightarrow \infty} d\left(f^{2 \mathscr{L}_{n}+p_{n}}\left(\hat{u}, t_{1}\right), f^{2 \mathscr{L}_{n}+p_{n}}\left(\hat{v}, t_{2}\right)\right)=0
$$

Therefore, $\left(\left(\hat{u}, t_{1}\right),\left(\hat{v}, t_{2}\right)\right) \in(U \times V) \cap \operatorname{LY}(f)$. Hence, $\left(\Sigma_{2} \times \mathbb{S}^{1}, f\right)$ is densely chaotic.

\section{Applying Theorem 8, the following can be verified.}

\section{Corollary 9 The following statements are equivalent:}

(1) $r_{1}-r_{0} \in \mathbb{R} \backslash \mathbb{Q}$;

(2) $\left(\Sigma_{2} \times \mathbb{S}^{1}, f\right)$ is densely chaotic;

(3) $\left(\Sigma_{2} \times \mathbb{S}^{1}, f\right)$ is densely $\delta$-chaotic for any $0<\delta<1$;

(4) $\left(\Sigma_{2} \times \mathbb{S}^{1}, f\right)$ is generically chaotic;

(5) $\left(\Sigma_{2} \times \mathbb{S}^{1}, f\right)$ is generically $\delta$-chaotic for any $0<\delta<1$.

\section{Acknowledgements}

Not applicable.

\section{Funding}

This work was supported by the MOE (Ministry of Education in China) Project of Humanities and Social Sciences (No. 19YJA790094), the Science and Technology Innovation Team of Education Department of Sichuan for Dynamical Systems and its Applications (No. 18TD0013), the Youth Science and Technology Innovation Team of Southwest Petroleum University for Nonlinear Systems (No. 2017CXTD02), the National Natural Science Foundation of China (No. 11601449 and 11701328) and the National Nature Science Foundation of China (Key Program) (No. 51534006).

\section{Availability of data and materials}

Data sharing not applicable to this article as no datasets were generated or analyzed during the current study.

\section{Competing interests}

The authors declare that they have no competing interests.

\section{Authors' contributions}

Each of the authors contributed equally to each part of this work. All authors read and approved the final manuscript.

\section{Publisher's Note}

Springer Nature remains neutral with regard to jurisdictional claims in published maps and institutional affiliations.

Received: 12 November 2018 Accepted: 31 July 2019 Published online: 03 September 2019

\section{References}

1. Akin, E., Kolyada, S.: Li-Yorke sensitivity. Nonlinearity 16, 1421-1433 (2003)

2. Li, J., Oprocha, P., Wu, X.: Furstenberg families, sensitivity and the space of probability measures. Nonlinearity 30 , 987-1005 (2017)

3. Li, J., Ye, X.: Recent development of chaos theory in topological dynamics. Acta Math. Sin. Engl. Ser. 32, 83-114 (2016)

4. Li, T.Y., Yorke, J.A.: Period three implies chaos. Am. Math. Mon. 82, 985-992 (1975)

5. Liao, G., Fan, Q.: Minimal subshifts which display Schweizer-Smítal chaos and have zero topological entropy. Sci. China Ser. A 41, 33-38 (1998)

6. Moothathu, T.K.S.: Stronger forms of sensitivity for dynamical systems. Nonlinearity 20, 2115-2126 (2007)

7. Oprocha, P., Wu, X.: On average tracing of periodic average pseudo orbits. Discrete Contin. Dyn. Syst. 37, 4943-4957 (2017)

8. Piórek, J.: On the generic chaos in dynamical systems. Univ. lagel. Acta Math. 25, 293-298 (1985)

9. Schweizer, B., Smítal, J.: Measures of chaos and a spectral decomposition of dynamical systems on the interval. Trans. Am. Math. Soc. 344, 737-754 (1994)

10. Sharkovsky, A.N.: Coexistence of cycles of a continuous map of a line into itself. Ukr. Mat. Zh. 16, 61-71 (1964) (Russian); English translation, Internat. J. Bifur. Chaos Appl. Sei. Engrg., 1995, vol. 5, pp. 1263-1273

11. Snoha, Ł.: Generic chaos. Comment. Math. Univ. Carol. 31, 793-810 (1990)

12. Snoha, Ł.: Dense chaos. Comment. Math. Univ. Carol. 33, 747-752 (1992)

13. Tan, F., Xiong, J.: Chaos via Furstenberg family couple. Topol. Appl. 156, 525-532 (2009)

14. Wang, H., Xiong, J., Tan, F.: Furstenberg families and sensitivity. Discrete Dyn. Nat. Soc. 2010, Article ID 649348 (2010). https://doi.org/10.1155/2010/649348

15. Wang, L., Liao, G., Chen, Z., Duan, X.: The set of recurrent points of a continuous self-map on compact metric spaces and strong chaos. Ann. Pol. Math. 82, 265-272 (2003) 
16. Wang, L., Liao, G., Yang, Y.: Recurrent point set of the shift on $\Sigma$ and strong chaos. Ann. Pol. Math. 78, 123-130 (2002)

17. $\mathrm{Wu}, \mathrm{X}$. : Chaos of transformations induced onto the space of probability measures. Int. J. Bifurc. Chaos 26, 1650227 (2016)

18. Wu, X.: A remark on topological sequence entropy. Int. J. Bifurc. Chaos 27, 1750107 (2017)

19. Wu, X., Chen, G.: Non-weakly almost periodic recurrent points and distributionally scrambled sets on $\Sigma_{2} \times \mathbb{S}^{1}$. Topol. Appl. 162, 91-99 (2014)

20. Wu, X., Ding, X., Lu, T., Wang, J.: Topological dynamics of Zadeh's extension on upper semi-continuous fuzzy sets. Int. J. Bifurc. Chaos 27, 1750165 (2017)

21. Wu, X., Liang, S., Luo, Y., Xin, M., Zhang, X.: A remark on the limit shadowing property for iterated function systems. UPB Sci. Bull., Ser. A 81, 107-114 (2019)

22. Wu, X., Luo, Y., Ma, X., Lu, T.: Rigidity and sensitivity on uniform spaces. Topol. Appl. 252, 145-157 (2019)

23. Wu, X., Luo, Y., Wang, L., Liang, J.: $\left(\mathscr{F}_{1}, \mathscr{F}_{2}\right)$-chaos and sensitivity for time-varying discrete systems. UPB Sci. Bull., Ser. A $81,153-160(2019)$

24. Wu, X., Ma, X., Zhu, Z., Lu, T.: Topological ergodic shadowing and chaos on uniform spaces. Int. J. Bifurc. Chaos 28 $1850043(2018)$

25. Wu, X., Oprocha, P., Chen, G.: On various definitions of shadowing with average error in tracing. Nonlinearity 29, 1942-1972 (2016)

26. Wu, X., Wang, J., Chen, G.: F -sensitivity and multi-sensitivity of hyperspatial dynamical systems. J. Math. Anal. Appl. $429,16-26(2015)$

27. Wu, X., Wang, X.: On the iteration properties of large deviations theorem. Int. J. Bifurc. Chaos 26, 1650054 (2016)

28. Wu, X., Wang, X., Chen, G.: On the large deviations theorem of weaker types. Int. J. Bifurc. Chaos 27, 1750127 (2017)

29. Wu, X., Zhang, X., Ma, X.: Various shadowing in linear dynamical systems. Int. J. Bifurc. Chaos 29, 1950042 (2019)

30. $Y$ e, $X$. ., Zhang, R.: On sensitivity sets in topological dynamics. Nonlinearity $21,1601-1620$ (2008)

31. Zhang, X., Wu, X., Luo, Y., Ma, X.: A remark on limit shadowing for hyperbolic iterated function systems. UPB Sci. Bull. Ser. A 81, 139-146 (2019)

\section{Submit your manuscript to a SpringerOpen ${ }^{\circ}$ journal and benefit from:}

- Convenient online submission

- Rigorous peer review

- Open access: articles freely available online

- High visibility within the field

- Retaining the copyright to your article

Submit your next manuscript at $>$ springeropen.com 